Western University

Scholarship@Western

Physics and Astronomy Publications

Physics and Astronomy Department

2013

\title{
Optimization of Gold Nanoring Arrays for Biosensing in the Fiber-optic Communication Window
}

Hao Jiang

Western University

Tingjie Li

Western University

Jun Yang

Western University

Silvia Mittler

Western University, smittler@uwo.ca

Jayshri Sabarinathan

Western University

Follow this and additional works at: https://ir.lib.uwo.ca/physicspub

Part of the Astrophysics and Astronomy Commons, and the Physics Commons

Citation of this paper:

Jiang, Hao; Li, Tingjie; Yang, Jun; Mittler, Silvia; and Sabarinathan, Jayshri, "Optimization of Gold Nanoring Arrays for Biosensing in the Fiber-optic Communication Window" (2013). Physics and Astronomy Publications. 41.

https://ir.lib.uwo.ca/physicspub/41 
Nanotechnology, 24 (46) (2013) 465502

\title{
Optimization of gold nanoring arrays for biosensing in the fiber-optic communication window
}

\author{
Hao Jiang ${ }^{1,4}$, Tingjie $\mathrm{Li}^{2,5}$, Jun Yang ${ }^{2}$, Silvia Mittler ${ }^{3}$ and Jayshri Sabarinathan ${ }^{1}$ \\ ${ }^{1}$ Department of Electrical and Computer Engineering, Western University, London, ON, Canada \\ 2 Department of Mechanical and Materials Engineering, Western University, London, ON, \\ Canada \\ ${ }^{3}$ Department of Physics and Astronomy, Western University, London, ON, Canada
}

\begin{abstract}
To improve the limit of detection in a nanoplasmonic sensor system, the optical performance of the metal nanostructures should be optimized according to the best spectral window of the measurement instrument. We propose that the spectral window from 1460 to $1610 \mathrm{~nm}$ can potentially provide ultrahigh instrumental resolution for biosensing. We optimized gold nanoring arrays such that the extinction peak position is inside the proposed window, the extinction peak is sharp enough to track the peak shift with high resolution and the figure of merit (sensitivity/linewidth) of the array is optimized at the same time. The peak-sharpening effect of the array caused by coherent interaction plays a central role in the optimization. The optimized array has a lattice constant in the range [1000 nm; $1060 \mathrm{~nm}]$, a bulk index sensitivity of around $450 \mathrm{~nm}=\mathrm{RIU}$ and a figure of merit larger than 4 . It is an enabling sensor element for a nearinfrared sensor chip with ultrahigh resolution.
\end{abstract}




\section{Introduction}

Label-free nanoplasmonic sensors based on metal nanoholes and nanoparticles allow for rapid and real-time detection of biomarkers and chemical stimuli [1-13]. Owing to the very small dimensions, many high-performance nanoplasmonic sensor elements can be compactly fabricated onto one sensor chip integrated with microfluidic channels to enable multiplexed detections [14, 15]. Such lab-on-chip devices hold the promise to meet the ever increasing demands of biological research and medical diagnostics $[10,16,17]$. For a nanoplasmonic sensor, the limit of detection (LOD) or resolution is mainly determined by the optical properties of metal nanostructures, the instrumental resolution and the surface chemistry. If one focuses on the physical aspects alone, in order to improve the limit of detection, the essential target is to increase the sensitivity and to reduce the detection uncertainty. The sensitivity is determined by the optical properties of the metal nanostructures and can be improved by adjusting the nanostructure compositions, geometries and substrate effects [18-26]. The sensitivity can also be improved by directing the analytes to highsensitivity areas [27-29]. For the sensors based on detecting peak shifts, a sharper peak with smaller linewidth can significantly reduce the uncertainty [25, 30, 31]. A figure of merit defined as the sensitivity divided by the linewidth can adequately be used to evaluate the optical properties of metal nanostructures for sensing applications [21, 25, 32, 33]. For a chosen type of nanostructure, the figure of merit can be improved by a few times by optimizing the structure parameters $[32,34,35]$.

The instrumental resolution of an optical measurement system is often a main factor in determining a sensor's limit of detection. By optimizing the signal-to-noise ratio of the instrument and the data analysis algorithm, the uncertainty can be significantly reduced by more than two orders of magnitude [36]. Chen et al have achieved a detection uncertainty in the order of $9.9 \times 10^{-5} \mathrm{~nm}$ and a limit of detection down to a few $\mathrm{pg} \mathrm{cm}^{-2}$ in sensing biomolecules using gold nanodisks of moderate sensitivity [9]. By engineering the sensor structure and the measurement system, Cervantes Tellez et al have reached a remarkable bulk sensing resolution of $9.38 \times 10^{-8}$ refractive index units (RIU) using atomically flat gold nanohole arrays, comparable to the conventional planar surface plasmon resonance sensors [26]. Considering all aforementioned aspects, an ideal sensor device could be described as having nanostructures with high figure of merit loaded into a 
low-noise measurement system. Therefore, the nanostructures should be optimized to function in the optimal spectral window of the measurement system. Although many measurement systems are sensitive over a very wide spectral range, the optimal spectral window with the best instrumental resolution is often narrow. Currently, most demonstrated sensors operated in the visible/near-infrared range below $1 \mu \mathrm{m}$ wavelength because the plasmon resonances are usually in that range. Another important spectral window, the fiber-optic communication window, has so far been overlooked for sensing applications. It has great potential for high resolution nanoplasmonic sensors because many optical components and devices are optimized for this window.

We propose a spectral window of 1460-1610 nm within the fiber-optic communication window for sensing applications. Such a window overlaps with the operating range of common tunable near-infrared lasers and the high responsivity range of InGaAs photodetectors. Water absorption peaks at 1200, 1450 and $1950 \mathrm{~nm}$ can also be avoided by this window. Nanoplasmonic sensors functioning in this window are compatible with silicon photonic platforms and can potentially be integrated with silicon-based micro-electronic and opto-mechanical components to enable novel applications. In this work, we studied gold nanoring arrays for biosensing in the proposed window. Gold nanorings have been demonstrated with widely tunable near-infrared plasmon resonance [37] and are promising candidates for index sensing [19, 34, 38, 39], surface-enhanced near-infrared Raman spectroscopy and absorption spectroscopy [40]. By engineering the coherent interaction of the gold nanoring arrays, we aimed to achieve a structure with plasmon peak position, peak linewidth and figure of merit all optimized at the same time. Such an optimization scheme on nanostructures considers not only the figure of merit of the nanostructure itself but also the best spectral window of the measurement instrument. Such an effort is the first step towards an ultrahigh resolution near-infrared sensor chip. The proposed spectral window is quite narrow and therefore puts a strong selection rule on the gold nanoring arrays. For tracking the peak position with high resolution, the portion of the extinction spectrum above the half-maximum should be measured [36]. Therefore, to function in the proposed spectral window, the extinction peak linewidth has to be reduced below $150 \mathrm{~nm}$. Such a narrow linewidth in near-infrared spectrum is challenging for single nanoparticles but can be readily achieved in a periodic array $[25,34,41-$ 50]. According to our previous study on gold nanoring arrays, as the lattice constant increases in the evanescent grating order range, the extinction peak significantly sharpens and the figure of 
merit is greatly improved [34]. The highest figure of merit of the array can be three times higher than that of single nanorings. The extinction peak position is tunable over a wide spectral range and the sensitivity decreases abnormally when the grating-induced modes begin to take effect. All these trends were implemented herein to optimize the nanoring arrays.

\section{Research methods}

The sensor configuration based on gold nanoring arrays is demonstrated in figure 1(a). Gold nanoring arrays of square lattice (lattice constant is denoted as 'a') are patterned on top of a pyrex wafer. Pyrex wafers were chosen for their excellent optical quality in the near-infrared range. The gold nanoring arrays were integrated in a transparent flow cell for introducing solutions and the extinction spectra were measured by normal transmission of near-infrared light. For biosensing, receptors are first immobilized onto the surface of the gold nanorings. When the analytes are introduced into the sensor, the receptors selectively capture the target biomolecules, due to the bioaffinity between the receptors and target biomolecules. As a result, the refractive index of the adlayer on the nanoring surface increases and the extinction peak red-shifts. The amount of the peak shift depends on the concentration of the target biomolecules.

\subsection{Sample fabrication}

The gold nanoring arrays in square lattice were fabricated on non-conductive pyrex substrates by electron beam lithography (EBL). The challenge is that the non-conductive glass substrate has to be made conductive for EBL purpose. In our previous study, we introduced a $25 \mathrm{~nm}$ thick indium tin oxide (ITO) conductive layer on top of the glass [34]. However, the ITO layer was found to broaden the extinction peak and decrease the sensitivity by more than $30 \%$, probably due to its lossy nature in the near-infrared spectral range. In this work, the problem was circumvented by depositing a sacrificial chromium layer on top of the photoresist prior to the EBL. After EBL, the chromium layer was removed by wet chemical etching. The details of the fabrication process are presented in the supplementary information. Figure 1(b) shows the scanning electron microscope 
(SEM) images of the fabricated structures. The geometries of the fabricated nanorings were acquired from SEM images by using an image processing software program. The center diameter of fabricated nanorings was around $235 \mathrm{~nm}$ and the wall thickness was around $60 \mathrm{~nm}$ with a standard deviation of $2.5 \mathrm{~nm}$.

\subsection{Measurement}

The extinction spectrum of each array was measured by the normal transmission of linearly polarized near-infrared broadband light $(1100-1700 \mathrm{~nm})$ focused by a 10 x objective $(\mathrm{NA}=0: 25)$. The focused beam spot (around $40 \mu \mathrm{m}$ in diameter) is much smaller than the size of the array to eliminate edge effects. Transmitted light was collected by a 20 x objective lens $(\mathrm{NA}=0: 2$, working distance $=20 \mathrm{~mm}$ ), coupled into a $0.8 \mathrm{~m}$ spectrograph and analyzed by an InGaAs detector. The extinction spectrum was calculated as $\operatorname{Ext} .(\lambda)=1-\operatorname{Idev} .(\lambda) / \operatorname{Iref} .(\lambda)$, with $\operatorname{Idev} .(\lambda)$ being the light transmitted through the nanoring arrays and Iref. $(\lambda)$ being the reference spectrum.

\subsection{Numerical simulations}

3D finite difference time domain (FDTD) simulations (FDTD Solutions, version 6, Lumerical Inc.) were carried out to simulate the nanoring arrays. Periodic boundaries were applied in the directions of periodicities, with the lateral size of simulation region equal to the corresponding lattice constant, and perfectly matched layers (PML) were applied onto other directions. A non-uniform mesh configuration was implemented with a grid size of $4 \mathrm{~nm}$ to mesh the gold nanorings [51]. A plane wave source propagated perpendicular to the plane of periodicity and the transmitted power through the nanoring was recorded by the power monitor. FDTD model with periodic boundaries actually simulates a perfectly infinite-sized periodic array of exactly identical nanorings. In comparison, the fabricated structures were finite-sized arrays, with inevitable minor lattice dislocations and variations of individual nanorings. To simulate the real fabricated structures in FDTD is currently impractical as the computational intensity will abruptly increase with the number of nanorings in the simulation region. This needs to be factored in when comparing the simulated and measured results. 
3. Optimize the gold nanoring arrays

\subsection{Plasmon peak position and linewidth of the gold nanoring arrays}

Figure 2 shows the measured and simulated extinction spectra of the periodic arrays in water for lattice constant from 600 to $1100 \mathrm{~nm}$. The measured spectra matched well with the simulations in

the peak positions and the trends. For each array, the measured extinction spectrum shows a broader peak with weaker amplitude than the simulated spectrum. We believe such a discrepancy is caused by the fabrication uncertainty. The fabricated nanorings are not identical and the variations reduce the strength of the coherent interaction in the array. A similar phenomenon has also been reported elsewhere [48, 52].

Figure 3 shows the peak wavelength $\lambda$ LSPR and peak linewidth fwhm (full-width at halfmaximum) versus the lattice constant. As the lattice constant increases, the extinction peak significantly red-shifts and narrows, due to the coherent interaction of the periodic array [34, 41, 42]. Let ares be the lattice constant for the maximal red-shift. ares can be approximated from the crossing point between the curve $\lambda \operatorname{LSPR}(a)$ and the critical grating constant line defined by $\lambda=$ nsuba, where nsub is the refractive index of the substrate. For $a<$ ares, the interaction of the array is through the evanescent grating order. In this range, as a increases and becomes close to ares, the scattered fields of neighboring particles are almost in-phase and are constructively added to each other. As a result, the optical fields in the array plane are greatly enhanced that causes the peak to red-shift. In the meanwhile, the plasmon decay time increases and the peak linewidth significantly decreases. For $\mathrm{a}>$ ares, the interaction is through the radiative grating order. This range is not appropriate for the sensor applications due to the complicated extinction spectral shape and nonlinear response versus refractive index [34]. Therefore, optimization of the nanoring arrays should be carried out in the range $\mathrm{a}<$ ares.

The experimental results showed that the peak can be tuned from 1310 to $1600 \mathrm{~nm}$ and the peak linewidth can vary from 384 to $78 \mathrm{~nm}$. Such a wide range tuning made it possible for the nanoring arrays to operate in the proposed spectral window. For tracking the peak shift, the portion of the extinction spectrum above half-maximum needs to be measured. For a given array, the range 
$\lambda \mathrm{LSPR}-\mathrm{fwhm} / 2<\lambda<\lambda \mathrm{LSPR}+\mathrm{fwhm} / 2$ marks its requested spectral window from the measurement system. Figure 4 demonstrates the functioning spectral window of each array. The proposed spectral window of $1460-1610 \mathrm{~nm}$ is marked as a horizontal shadowed region. According to this figure, gold nanoring arrays with lattice constants $1000 \mathrm{~nm}<\mathrm{a}<1060 \mathrm{~nm}$ can function in the proposed spectral window.

\subsection{Sensitivity and figure of merit}

The bulk index sensitivity is defined as $\mathrm{mB}=\delta \lambda \mathrm{LSPR}=\delta \mathrm{nB}$, with $\mathrm{nB}$ being the bulk refractive index and the figure of merit is defined as $\mathrm{FOM}=\mathrm{mB} / \mathrm{fwhm}$. In order to measure the bulk index sensitivity, different water and isopropanol (IPA) mixtures were injected onto the sensor to change nB. Figure 5(a) shows the linear relationships of the peak shift versus the refractive index and the sensitivity of each array was determined from the slope. Similarly, in FDTD simulations, $\mathrm{nB}=$ $1.33,1.35$ and 1.37 were simulated for each array to find the sensitivity of nanoring arrays in water. Figure 5 show the bulk index sensitivity and figure of merit of each nanoring array. The measured sensitivity curve matched well with the simulated one in the range a $\leq 900 \mathrm{~nm}$. For a $>900 \mathrm{~nm}$, the measured sensitivity decreases less rapidly than the simulations. The abnormal decrease of the sensitivity is due to the coherent interaction of the array: when a is close to ares, the coherent interaction leads to an enhanced field in the substrate but a decreased field confinement in the sensing region [34]. Considering the fabrication uncertainty that reduces the coherent interaction, the decrease of sensitivity in experiments should not be as significant as in simulations.

As a increases, the decrease of the peak linewidth is much more pronounced than the change in sensitivity and the figure of merit is therefore significantly improved [34]. The experimental results show that FOMB increases from 1.4 into 5.1.

\subsection{Optimized gold nanoring arrays}

The optimized lattice constant lies in the range $1000 \mathrm{~nm}<\mathrm{a}<1060 \mathrm{~nm}$. Gold nanoring arrays of this range satisfy all the optimization targets: the plasmon peak is within the proposed spectral window, the peak linewidth is smaller than the window span and the figure of merit is among the 
highest values. For the array with a D $1000 \mathrm{~nm}$, the obtained bulk index sensitivity is around 457 $\mathrm{nm}=\mathrm{RIU}$ and the figure of merit is around 4.2 . For the array with a D $1050 \mathrm{~nm}$, the sensitivity is around $448 \mathrm{~nm}=\mathrm{RIU}$ and the figure of merit is around 4.9.

According to a recent review by Mayer et al summarizing the figure of merit of metal nanoparticles [11], our optimized gold nanoring arrays have a figure of merit comparable to those highest values.

\section{Discussions}

In optimizing the gold nanoring arrays, we first fixed the geometry of the nanoring to be center diameter of $235 \mathrm{~nm}$, wall thickness of $60 \mathrm{~nm}$ and height of $50 \mathrm{~nm}$. It should be clarified that this geometry was carefully chosen according to the numerical simulations. Different sizes of gold nanorings were first simulated. Specifically, we have simulated the nanorings with center diameters spanning the range $[215 \mathrm{~nm}, 300 \mathrm{~nm}]$ and wall thickness spanning the range [35 nm, $100 \mathrm{~nm}]$. Out of the simulation results, the chosen nanoring geometry is the best candidate to make the sensor function as desired. If we compare the plasmon peak of a single nanoring and the optimized array constructed with this nanoring, the array often shows a plasmon peak wavelength longer than the single nanoring by $60-100 \mathrm{~nm}$. This can roughly serve as an empirical rule to select the gold nanoring based on the expected spectral window of the optimized array. For example, if the optimized array is expected to function close to the wavelength $1300 \mathrm{~nm}$, the single nanoring used to construct the array should probably show a resonance in the range [1200 nm, $1240 \mathrm{~nm}$ ]. As the lattice constant increases in the evanescent grating order range $(a<$ ares), the sensitivity decreases, because the optical fields are greatly enhanced in the substrate which reduces the field confinement factor and sensitivity. The sensitivity of the nanoring arrays also depends on the wall thickness, diameter and height. Considering all these parameters, it is difficult to describe a trend for all nanoring arrays. If one considers the various nanoring arrays with resonance peak in the same range and that the lattice constant remains in the evanescent grating order range, the change of sensitivity follows qualitative trends versus wall thickness and height. According to the study by Tsai et al, the sensitivity slightly increases with increasing wall thickness [39]. From our simulations, we found the sensitivity decreases with increasing height. 
In our previous work, we were not able to achieve a figure of merit larger than 1.43 , due to the decreased sensitivity and broadened peak caused by the ITO-coated substrate [34]. The highest figure of merit achieved from this work is 3 times larger and validates the sensing potential of the gold nanoring arrays. Single gold nanorings on pyrex substrate have been reported with a figure of merit around 2 [19]. The comparison suggests that the gold nanoring arrays show better sensing potential than the single nanorings. The peak-sharpening effect of the periodic array plays a central role in both improving the figure of merit and tailoring the extinction peak within the proposed narrow spectral window. The relatively sharp peaks may also open new possibilities for multiplexed detections, for example, the sharp peaks of different sensor elements may be differentiated from a single measurement of all sensor elements simultaneously.

To prove the biosensing applications, we implemented the optimized gold nanoring array with $\mathrm{a}=$ $1000 \mathrm{~nm}$ to detect biotin molecules binding to the streptavidins on the sensor surface. To immobilize the streptavidins, we followed the procedures described elsewhere [25, 53]. The biochemistry procedures are presented in the supplementary information. Figure 6 shows the peak shift versus biotin concentrations. In the current detection scheme, a halogen lamp was used as the light source and the extinction spectra were only recorded in the spectral window 1460-1610 nm. The sensor proved to function correctly in this window and the uncertainty of peak shift was around $0.03 \mathrm{~nm}$. As the biotin concentration increases from $1 \mu \mathrm{g} \mathrm{ml}^{-1}$ to $1 \mathrm{mg} \mathrm{ml}^{-1}$, the peak red-shifts by around $0.45 \mathrm{~nm}$. The limit of detection for biotin was better than $1 \mu \mathrm{g} \mathrm{ml}^{-1}$ and the refractive index resolution was around $2 \times 10^{-4}$ RIU. The binding of the biotin molecules with streptavidins on the sensor surface follows the Langmuir adsorption kinetics and the sensor response versus concentration is usually a dose response curve $[54,55]$.

At very low concentration, the sensor response is in linear relationship with concentration. For larger concentrations, such as the range of biotin concentration tested in our experiment, the sensor response is almost proportional to the logarithmic of the concentration. Since the sensor structure has been optimized to function in the 1460-1610 nm window, the measurement system can take advantage of mature technologies developed for fiber-optic communication and silicon photonics. For example, once a near-infrared tunable laser is implemented as the light source, the intense stable laser light can substantially reduce the noise. The rapid scan of the wavelengths will also 
allow the spectra to be measured with high spectral resolution in real-time. The instrumental resolution can be improved by several orders of magnitude and ultrahigh sensor resolution can thus be achieved.

Although the main scope of this work is label-free index sensing using the nanoring arrays, the presented nanoring array structures could also be potentially used in other applications, such as mapping the cells with sub-cellular resolution [56].

\section{Summary}

We have successfully optimized gold nanoring arrays for biosensing applications in the proposed spectral window of 1460-1610 nm. The optimized array has a lattice constant in the range 1000 $\mathrm{nm}<\mathrm{a}<1060 \mathrm{~nm}$, with a sensitivity around $450 \mathrm{~nm}=$ RIU and figure of merit larger than 4 . The extinction peak linewidth is narrow enough to allow the peak shift be tracked with high resolution. The sensing characteristics

of gold nanoring arrays found in experiments matched qualitatively with FDTD simulations. The differences in quantities were attributed to the strength of the coherent interaction in the array.

\section{Acknowledgments}

The authors acknowledge Natural Sciences and Engineering Research Council of Canada (NSERC), CMC Microsystems and Canada Foundation for Innovation (CFI) for funding this research work. The device fabrication was done at the University of Western Ontario Nanofabrication facility. The FDTD simulations were carried out using SHARCNET computing facilities. The authors acknowledge student

funding for H Jiang from NSERC: the BiopSys Network. S Mittler likes to thank the Canadian Government for the CRC program. 


\section{References}

[1] Englebienne P 1998 Analyst 123 1599-603

[2] McFarland A D and Van Duyne R P 2003 Nano Lett. 3 1057-62

[3] Brolo A G, Gordon R, Leathem B and Kavanagh K L 2004 Langmuir 20 4813-5

[4] Willets K A and Van Duyne R P 2007 Annu. Rev. Phys. Chem. 58 267-97

[5] Mack N H, Wackerly J W, Malyarchuk V, Rogers J A, Moore J S and Nuzzo R G 2007 Nano Lett. 7 733-7

[6] Anker J N, Hall W P, Lyandres O, Shah N C, Zhao J and Van Duyne R P 2008 Nature Mater. $7442-53$

[7] Stewart M E, Anderton C R, Thompson L B, Maria J, Gray S K, Rogers J A and Nuzzo R G 2008 Chem. Rev.108 494-521

[8] Jiang H, Markowski J and Sabarinathan J 2009 Opt. Express 17 21802-7

[9] Chen S, Svedendahl M, K“all M, Gunnarsson L and Dmitriev A 2009 Nanotechnology 20 434015

[10] Yanik A A, Huang M, Kamohara O, Artar A, Geisbert T W, Connor J H and Altug H 2010 Nano Lett. 10 4962-9

[11] Mayer K M and Hafner J H 2011 Chem. Rev. 111 3828-57

[12] Brolo A G 2012 Nature Photon. 6 709-13

[13] Jia P, Jiang H, Sabarinathan J and Yang J 2013 Nanotechnology 24195501

[14] Yu C and Irudayaraj J 2007 Anal. Chem. 79 572-9

[15] Endo T, Kerman K, Nagatani N, Hiepa H M, Kim D K, Yonezawa Y, Nakano K and Tamiya E 2006 Anal. Chem.

$786465-75$

[16] Huang C, Bonroy K, Reekman G, Verstreken K, Lagae L and Borghs G 2009 Microelectron. Eng. $862437-41$

[17] Escobedo C, Chou Y W, Rahman M, Duan X, Gordon R, Sinton D, Brolo A G and Ferreira J 2013 Analyst 138 1450-8

[18] Dmitriev A, H“agglund C, Chen S, Fredriksson H, Pakizeh T, K“all M and Sutherland D S 2008 Nano Lett. 8 3893-8

[19] Larsson E M, Alegret J, K“"all M and Sutherland D S 2007 Nano Lett. 7 1256-63 
[20] Novo C, Funston A M, Pastoriza-Santos I, Liz-Marzan L M and Mulvaney P 2008 J. Phys. Chem. C 112 3-7

[21] Sherry L J, Chang S H, Schatz G C, Van Duyne R P, Wiley B J and Xia Y 2005 Nano Lett. 5 2034-8

[22] Murray W A, Augui'e B and Barnes W L 2009 J. Phys. Chem. C 113 5120-5

[23] Najiminaini M, Vasefi F, Kaminska B and Carson J J 2012 Appl. Phys. Lett. 100063110

[24] Monteiro J P, Carneiro L B, Rahman M M, Brolo A G, Santos M J, Ferreira J and Girotto E M 2013 Sensors Actuators B 178 166-70

[25] Jiang H, Li T, Ertorer E, Yang J, Sabarinathan J and Mittler S 2013 Sensors Actuators A 189 474-80

[26] Cervantes Tellez G A, Hassan S, Tait R N, Berini P and Gordon R 2013 Lab Chip 13 25416

[27] Eftekhari F, Escobedo C, Ferreira J, Duan X, Girotto E M, Brolo A G, Gordon R and Sinton D 2009 Anal. Chem. 81 4308-11

[28] Escobedo C, Brolo A G, Gordon R and Sinton D 2010 Anal. Chem. 82 10015-20

[29] Feuz L, J“onsson P, Jonsson M P and H“o”ok F 2010 ACS Nano 4 2167-77

[30] Unger A and Kreiter M 2009 J. Phys. Chem. C 113 12243-51

[31] Nusz G J, Curry A C, Marinakos S M, Wax A and Chilkoti A 2009 ACS Nano 3 795-806

[32] Otte M A, Sep'ulveda B, Ni W, Juste J P, Liz-Marz'an L M and Lechuga L M 2010 ACS Nano 4 349-57

[33] Tetz K A, Pang L and Fainman Y 2006 Opt. Lett. 31 1528-30

[34] Jiang H and Sabarinathan J 2010 J. Phys. Chem. C 114 15243-50

[35] Jiang H, Sabarinathan J and Mittler S 2012 J. Phys. Chem. C 11612375

[36] Dahlin A B, Tegenfeldt J O and H“o”ok F 2006 Anal. Chem. 78 4416-23

[37] Aizpurua J, Hanarp P, Sutherland D S, K“all M, Bryant G W and Garcia de Abajo F J 2003 Phys. Rev. Lett. 90057401

[38] Liu S D, Zhang Z S and Wang Q Q 2009 Opt. Express 17 2906-17

[39] Tsai C Y, Lu S P, Lin J W and Lee P T 2011 Appl. Phys. Lett. 98153108

[40] Teo S L, Lin V K, Marty R, Large N, Llado E A, Arbouet A, Girard C, Aizpurua J, Tripathy S and Mlayah A 2010 Opt. Express 18 22271-82

[41] Meier M, Wokaun A and Liao P F 1985 J. Opt. Soc. Am. B 2 931-49 
[42] Lamprecht B, Schider G, Lechner R T, Ditlbacher H, Krenn J R, Leitner A and Aussenegg F R 2000 Phys. Rev. Lett. 84 4721-4

[43] Sung J, Hicks E M, Van Duyne R P and Spears K G 2007 J. Phys. Chem. C 111 10368-76

[44] Sung J, Hicks E M, Van Duyne R P and Spears K G 2008 J. Phys. Chem. C 112 4091-6

[45] Haynes C L, McFarland A D, Zhao L, Van Duyne R P, Schatz G C, Gunnarsson L, Prikulis

J, Kasemo B and K"all M 2003 J. Phys. Chem. B 107 7337-42

[46] Zou S, Janel N and Schatz G C 2004 J. Chem. Phys. 120 10871-5

[47] Zou S and Schatz G C 2004 J. Chem. Phys. 121 12606-12

[48] Hicks E M, Zou S, Schatz G C, Spears K G, Van Duyne R P, Gunnarsson L, Rindzevicius T, Kasemo B and Kall M 2005 Nano Lett. 5 1065-70

[49] Nikitin A G, Kabashin A V and Dallaporta H 2012 Opt. Express 20 27941-52

[50] Augui'e B and Barnes W L 2008 Phys. Rev. Lett. 101143902

[51] Jiang H, Sabarinathan J, Manifar T and Mittler S 2009 J. Lightwave Technol. 27 2264-70

[52] Augui'e B and Barnes W L 2009 Opt. Lett. 34 401-3

[53] Choi S H, Lee J W and Sim S J 2005 Biosens. Bioelectron. 21 378-83

[54] Chen C D, Cheng S F, Chau L K and Wang C 2007 Biosens. Bioelectron. 22 926-32

[55] Marinakos S M, Chen S and Chilkoti A 2007 Anal. Chem. 79 5278-83

[56] Wang S et al 2011 Nano Lett. 11 3431-4 


\section{Figure captions:}

Figure 1. Gold nanoring arrays for sensing applications. (a) Schematic of the sensor configuration. (b) SEM images of the fabricated nanorings (square lattice) with lattice constants from 600 to 1100 $\mathrm{nm}$, with a step of $100 \mathrm{~nm}$. The sample has been coated with a $3 \mathrm{~nm}$ osmium layer prior to SEM imaging.

Figure 2. The extinction spectra of gold nanoring arrays in water: (a) experimental and (b) simulation results. The text and arrow in the figures denote the lattice constant and the direction of the peak shift. As the lattice constant increases from 600 to $1100 \mathrm{~nm}$, with a step of $50 \mathrm{~nm}$, the peak red-shifts.

Figure 3. Spectral characteristics of gold nanoring arrays in water. (a) Measured and simulated extinction peak wavelength. (b) Measured and simulated linewidth fwhm. The solid line in (a) represents the critical grating constant versus wavelength. For peaks located above the line, the interaction of the array is through evanescent grating orders. For the peaks located below the line, the interaction is through radiative grating orders.

Figure 4. Determine the functioning spectral window for each array according to the experimental results. The proposed spectral window of 1460-1610 nm and the appropriate lattice constants 1000 $\mathrm{nm}<\mathrm{a}<1060 \mathrm{~nm}$ are marked by the shadow regions.

Figure 5. The sensitivity and figure of merit of the gold nanoring arrays. (a) Measured $\lambda$ LSPR versus bulk refractive index, for $\mathrm{a}=1000,1050$ and $1100 \mathrm{~nm}$. The solid symbols are measured peak wavelength and the solid lines are the corresponding linear fits. (b) Measured and simulated bulk index sensitivity. (c) Measured and simulated figure of merit.

Figure 6. Sensor response of array with lattice constant $a=1000 \mathrm{~nm}$ : peak shift versus biotin concentrations. The straight line serves as a guide to the eye. 
Figures:

FIG.1

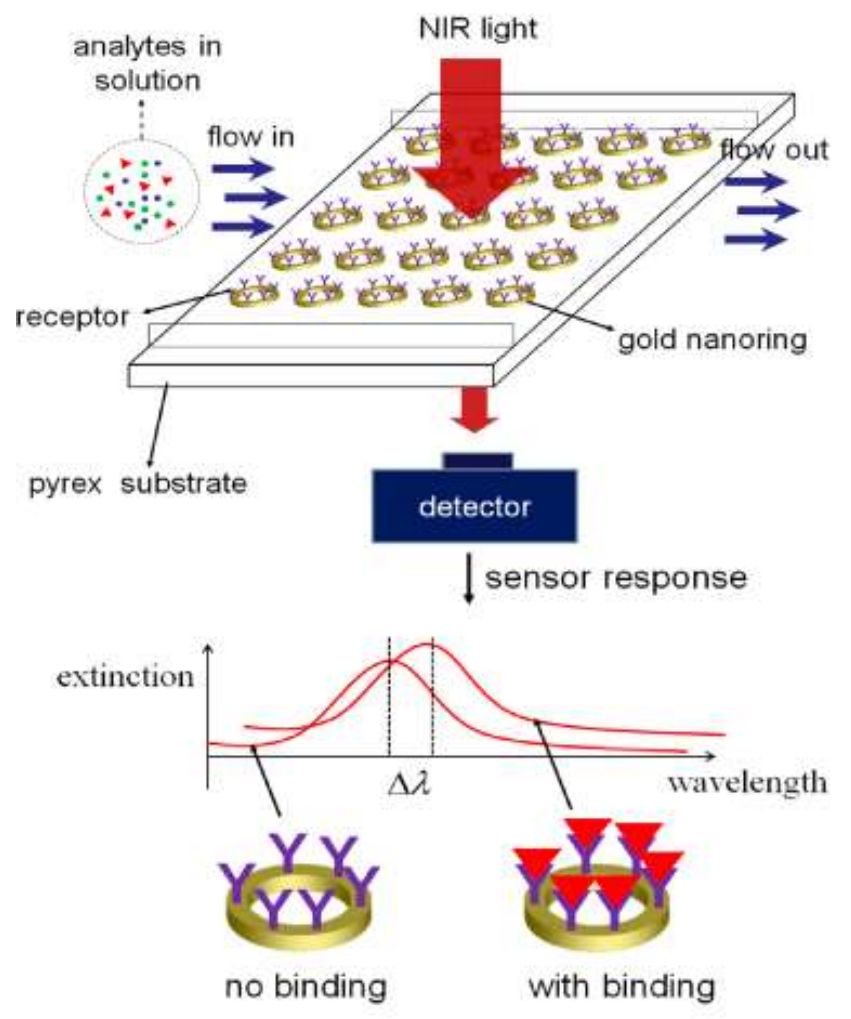

(a)
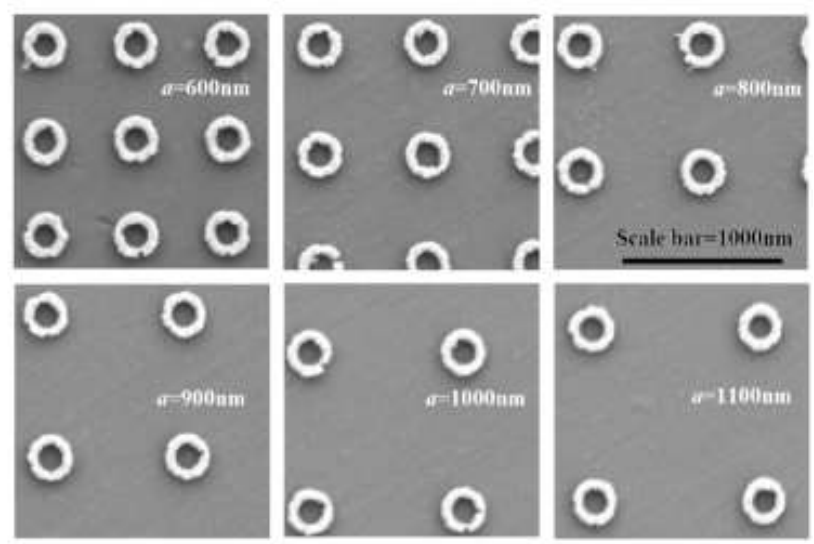

(b) 
FIG.2

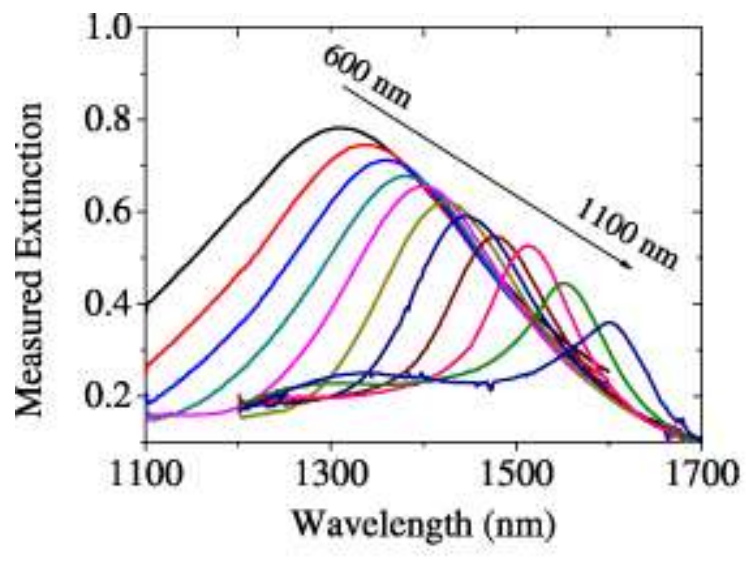

(a)

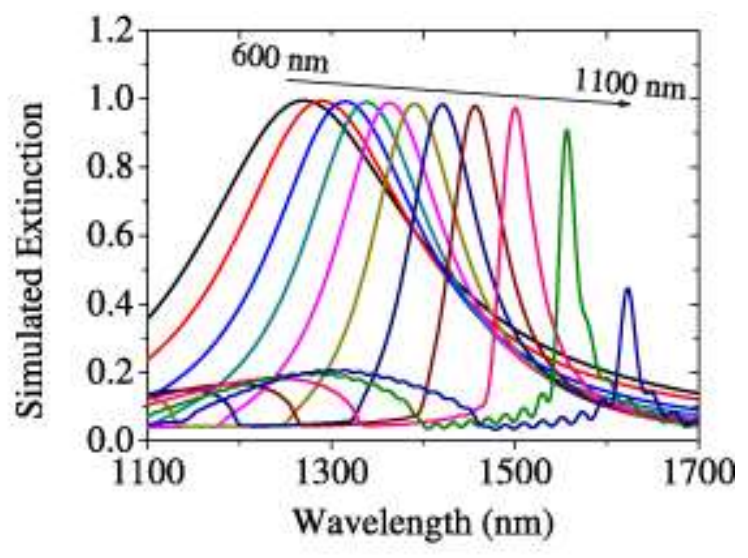

(b)

FIG.3

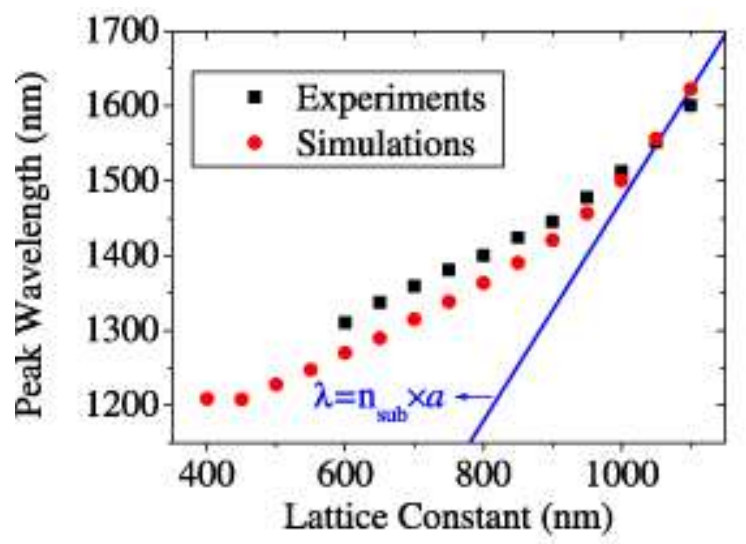

(a)

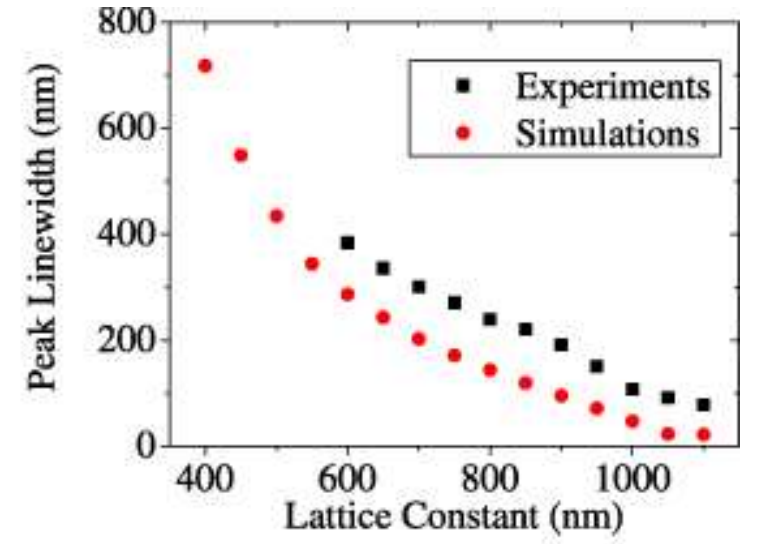

(b) 
FIG.4

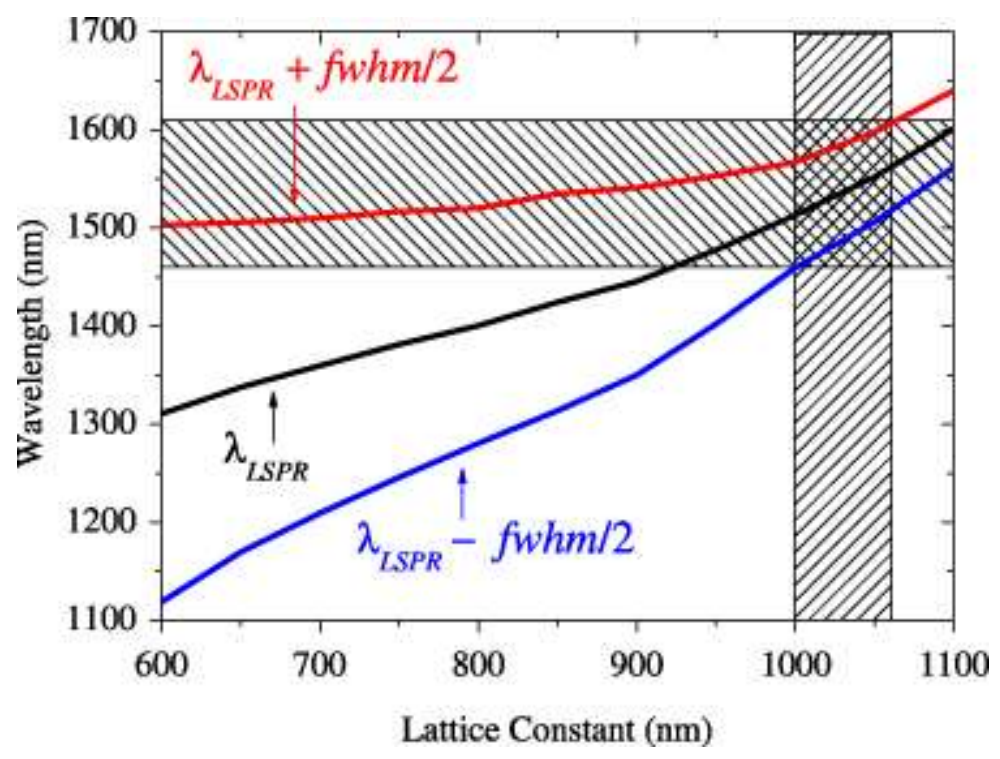

FIG.5

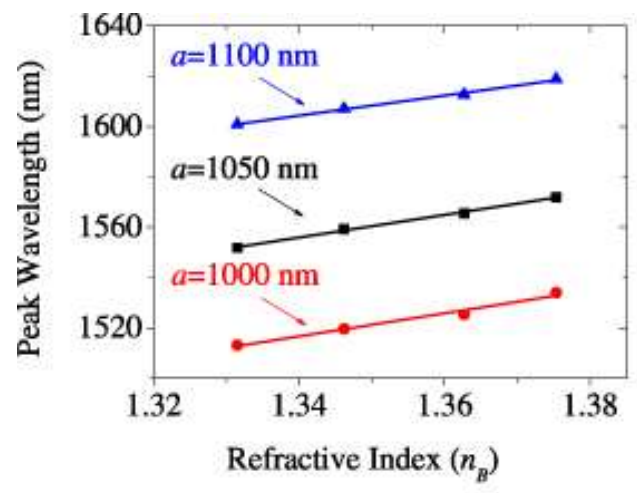

(a)

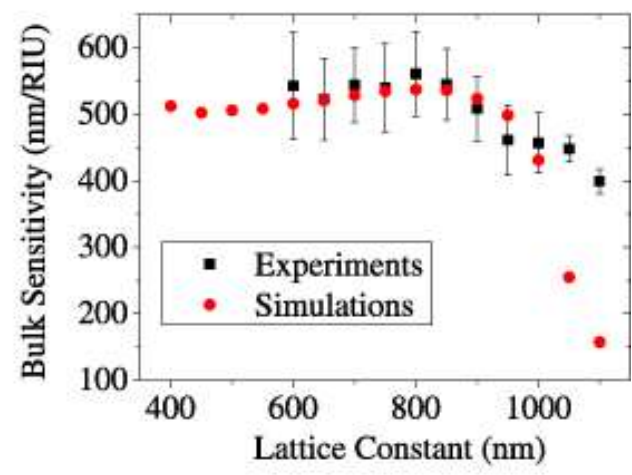

(b)

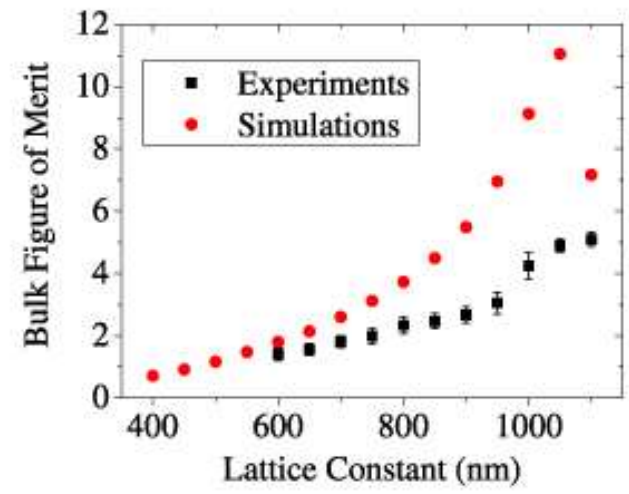

(c) 
FIG.6

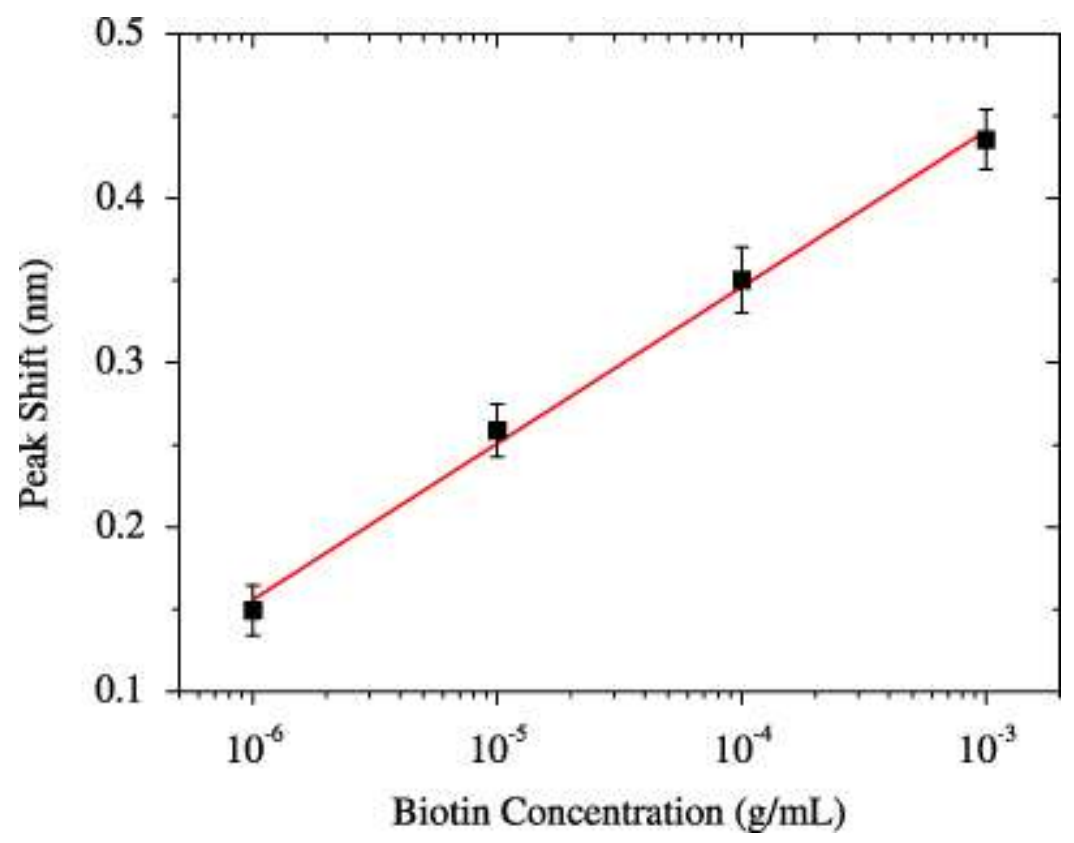

